\title{
Impaired ventilatory function and elevated insulin levels in nondiabetic males: the Normative Aging Study
}

\author{
R. Lazarus*+, D. Sparrow ${ }^{\ddagger}$, S.T. Weiss ${ }^{+}$
}

Impaired ventilatory function and elevated insulin levels in nondiabetic males: the Normative Aging Study. R. Lazarus, D. Sparrow, S.T. Weiss. CERS Journals Ltd 1998.

ABSTRACT: Lower levels of baseline ventilatory function have consistently been associated with increased risk of cardiovascular mortality in prospective studies, but the underlying mechanisms are not known. Increased risk of coronary heart disease is associated with higher serum insulin levels. This report examines the relationship between ventilatory function and indirect measures of insulin resistance.

Cross-sectional data from 922 nondiabetic participants in the Normative Aging Study were analysed using multiple linear regression models with adjustment for potential confounders. Forced vital capacity (FVC) and forced expiratory volume in one second (FEV1) were examined in relation to indicators of insulin resistance, i.e. fasting insulin and the fasting insulin resistance index (FIRI). Diabetics were excluded because impaired insulin secretion interferes with the validity of these as measures of insulin resistance.

Fasting insulin and FIRI were negatively correlated with FVC and FEV1 (all p< 0.001). These associations persisted after adjusting for potential confounders including age, height, body mass index, waist to hip circumference ratio, physical activity, alcohol intake and smoking in separate multiple linear regression models, for both insulin (all pð0.0008) and FIRI (all pð0.0001).

Negative cross-sectional associations between ventilatory function and indirect measures of insulin resistance were found in nondiabetic males. Insulin resistance may contribute to the previously unexplained association between ventilatory function impairment and cardiovascular mortality. Mechanisms underlying the relationship between insulin resistance and decreased ventilatory function remain to be elucidated.

Eur Respir J 1998; 12: 635-640.
*Dept of Public Health and Community Medicine, Faculty of Medicine, University of Sydney, Australia. ${ }^{+}$Channing Laboratory, Brigham and Women's Hospital, Harvard Medical School, Boston, USA. "Normative Aging Study, Dept of Veterans Affairs, Boston, USA.

Correspondence: R. Lazarus, Dept of Public Health and Community Medicine, Westmead Hospital, Westmead, NSW 2145, Australia. Fax: 61296891049

Keywords: Coronary disease, insulin resistance, spirometry

Received: July 71997

Accepted after revision March 71998

Supported by grants HL-37871 and HL45089 from the National Heart Lung and Blood Institute, grant MO1RR01032 from the General Clinical Research Center, Beth Israel Hospital and the Health Services Research and Development Service of the Dept of Veterans Affairs. D. Sparrow is an Associate Career Scientist from the VA Medical Research Service. R. Lazarus was supported by a Special Studies Program from the University of Sydney.
A number of prospective population studies have found higher risk of cardiovascular mortality [1-3] and myocardial infarction $[1,4]$ among individuals with lower levels of baseline ventilatory function. Although several potential mechanisms have been proposed [5] this increased risk remains largely unexplained.

Pulmonary impairment is known to occur in both insulin-dependent diabetes mellitus (IDDM) $[6,7]$ and noninsulin-dependent diabetes mellitus (NIDDM) [8]. Glucose intolerance is a major risk factor for the development of NIDDM. Several reports have suggested that glucose intolerance is also associated with impaired ventilatory function [9-11]. In nondiabetic subjects, an inverse relationship between fasting plasma glucose and both forced vital capacity (FVC) and forced expiratory volume in one second (FEV1) was reported among males from the Rancho Bernardo cohort, but not among females [12].

The fundamental pathophysiological mechanism which is generally thought to precede the development of detectable glucose intolerance is resistance to the glucose regulatory actions of insulin (insulin resistance) [13, 14]. In the early stages of the natural history of NIDDM, elevated insulin levels may enable essentially normal glucose tolerance to be maintained. Eventually, glucose intolerance and NIDDM are thought to supervene as diminished insulin secretory capacity arises because of failure of the insulinproducing cells of the pancreas $[14,15]$. Thus, elevated insulin levels among apparently healthy subjects are a marker of insulin resistance [16] and may indicate increased risk for the development of glucose intolerance and NIDDM.

Substantially increased risk of incident coronary heart disease has been observed among males with higher serum insulin levels [17] and the association of cardiovascular disease risk with insulin-resistant states, including glucose intolerance and NIDDM [18], raises the possibility that insulin resistance might be one of the mechanisms underlying the prospective relationship between baseline ventilatory function and cardiovascular risk. Evidence of an inverse association between ventilatory function and insulin levels in nondiabetic subjects would support this hypothesis. To date, there have been relatively few published data on the relationship between ventilatory function and measures of either insulin or insulin resistance in nondiabetic subjects.

The purpose of this report was to examine the relationship between indirect measures of insulin resistance and ventilatory function in cross-sectional data from a relatively large group of nondiabetic males. It was hypothesized that higher levels of insulin and insulin resistance might be associated with decreased ventilatory function. 


\section{Methods}

\section{Subjects and measurements}

The Normative Aging Study (NAS) is an ongoing longitudinal study established by the United States Veterans Administration in 1961, which has been described in detail previously [19]. Volunteers were screened according to specific clinical, laboratory, spirometric, radiological, electrocardiographic and medical history criteria [19] at enrolment into the study cohort, which comprised 2,280 healthy community-dwelling males from the Boston area, aged $21-80$ yrs at entry.

Anthropometry was performed in accordance with the study protocol. Measurements were taken with the subject standing erect with feet together, clothed in undershorts and socks only. Weight in pounds was measured on a beam balance and converted into kilograms. Stature in metres was measured against a wall chart. Body mass index (BMI) [20] was calculated as the weight in kilograms divided by the square of the height in metres $\left(\mathrm{kg} \cdot \mathrm{m}^{-2}\right)$. The circumferences of the waist and hips were measured with a cloth tape measure and the ratio of waist circumference to hip circumference, or waist/hip ratio (WHR), was used as a measure of a central pattern of fat distribution. An estimate of usual physical activity level was made using a self-completed questionnaire [21]. Average daily alcohol intake was estimated from a semiquantitative food frequency questionnaire [22, 23].

Subjects performed spirometry in the standing position, wearing noseclips. A water-filled survey spirometer (Collins 8-L; Warren E. Collins, Braintree, MA, USA) was used in accordance with published American Thoracic Society (ATS) guidelines [24]. FVC (L) and FEV1 (L), corrected to body temperature and pressure saturated with water vap-our (BTPS), were calculated by a microprocessor (Eagle II; Warren E. Collins) attached to the spirometer. Detailed records of smoking history were recorded at each pulmonary function testing visit.

Subjects fasted overnight before their examination. Fasting blood samples were drawn and a glucose challenge of $100 \mathrm{~g}$ was administered orally. There is little difference in the results obtained by using a $100 \mathrm{~g}$ glucose load compared with the now more widely used $75 \mathrm{~g}$ [25]. Two hours later, postglucose challenge (PC) blood samples were drawn. For a limited period (from February 1987 until July 1991), insulin levels from fasting and PC blood samples were measured in addition to fasting and PC glucose. The blood insulin data from this limited period form the basis of this report and only subjects who were examined during this period were able to contribute data for analysis. Insulin was measured using a solid-phase ${ }^{125 I}$ radioimmunoassay (Coat-A-Count Insulin 1987; Diagnostic Products Corporation, Los Angeles, CA, USA). The interassay and intra-assay coefficients of variation were 5-7\% and $3-5 \%$, respectively.

The fasting insulin resistance index (FIRI) was used as an indirect measure of insulin resistance [26] and was calculated as the product of the fasting insulin level and the fasting glucose level. As recommended [26], it was normalized to a mean value of 1.0 by dividing by the product of the mean fasting insulin and fasting glucose values for the nondiabetic subjects (425.5).

\section{Exclusions}

A total of 1,223 ongoing NAS participants were examined during the period when blood insulin was being measured. Any second or subsequent record where a subject was examined more than once within the period was ignored. Of the subjects with insulin measurements available, 77 were excluded on the basis of a physician's diagnosis of diabetes mellitus in the past (of whom 34 were taking antidiabetic medication) and an additional 67 were excluded because they satisfied World Health Organization (WHO) criteria for diabetes mellitus (PC glucose $>11.1$ $\mathrm{mmol} \cdot \mathrm{L}^{-1}$ or fasting glucose $\left.>7.77 \mathrm{mmol} \cdot \mathrm{L}^{-1}\right)$ on one or more occasions at or before the visit when insulin was measured. An additional 157 subjects had missing values for one or more of the measurements needed, leaving a total of 922 nondiabetic subjects with suitable data available for the analysis.

Diabetic subjects were excluded because their insulin and glucose levels may have been influenced by treatment for their disease and because the indirect measures of insulin resistance available for this study are unreliable among diabetics, whose insulin secretory capacity may be impaired [16, 27].

\section{Analysis}

Spearman rank correlation coefficients were estimated between the study variables and potential confounders including age, BMI, WHR, lifetime total cigarette consumption (pack-yrs), usual physical activity and alcohol intake. Normal probability plots showed that the skewed distributions of FIRI and fasting insulin values were improved by a logarithmic transformation, so this was used in all parametric analyses.

Analysis of variance (ANOVA) was used to test for differences in continuous variables between fasting insulin tertile categories. FVC and FEV1 were adjusted for age and stature by including age and height in the regression models, because these are major determinants [28]. In addition, smoking [29], body fat distribution, obesity [30] and physical activity [13] are thought to be associated with insulin levels, so these were also included in models to adjust for their effects. Finally, a binary indicator term for pre-existing heart disease (physician's diagnosis of congestive cardiac failure, angina or myocardial infarction) at or before spirometry was used because pre-existing heart disease has resulted in impaired ventilatory function.

Multiple linear regression models were used to examine the association between FIRI and fasting insulin (as indicators of insulin resistance) and ventilatory function. Separate regression models with FVC and FEV1 as the outcome variables, with FIRI and fasting insulin as predictor variables, were examined in turn. These models were first tested with adjustment for age, height, pack-yrs of smo-king and current smoking status. The final models shown included additional adjustment for usual physical activity level, usual alcohol intake, BMI, WHR and preexisting heart disease. Least mean square adjusted FVC by tertile of fasting insulin was estimated using a generalized linear model with adjustment for all potential confounders. 
Table 1. - Characteristics of subjects by fasting insulin tertile and all combined (Normative Aging Study, 1987-1991)

\begin{tabular}{|c|c|c|c|c|}
\hline & $\begin{array}{c}\text { Low } \\
(n=307)\end{array}$ & $\begin{array}{l}\text { Intermediate } \\
(\mathrm{n}=304)\end{array}$ & $\underset{(n=311)}{\text { High }}$ & $\begin{array}{l}\text { All combined } \\
\quad(n=922)\end{array}$ \\
\hline Fasting insulin $\mathrm{pmol} \cdot \mathrm{L}^{-1+* * *}$ & $5.5 \quad(1.2)$ & $9.1 \quad(1.2)$ & $18.3(7.3)$ & $11.0 \quad(6.9)$ \\
\hline $\mathrm{FIRI}^{+* * *}$ & $0.50(0.12)$ & $0.86(0.15)$ & $1.77(0.78)$ & $1.00(0.71)$ \\
\hline Age yrs* & $63.0 \quad(7.8)$ & $64.4 \quad(7.7)$ & $62.4 \quad(7.5)$ & $63.3(7.7)$ \\
\hline Height $\mathrm{m}$ & $1.74(0.06)$ & $1.73(0.07)$ & $1.74(0.06)$ & $1.74(0.07)$ \\
\hline BMI kg·m-2+*** & $25.3 \quad(2.6)$ & $26.9 \quad(3.0)$ & $28.8 \quad(3.5)$ & $27.0 \quad(3.4)$ \\
\hline Waist/hip ratio*** & $0.96(0.05)$ & $0.98(0.04)$ & $1.0 \quad(0.05)$ & $0.98(0.05)$ \\
\hline $\mathrm{FVC} \mathrm{L***}$ & $4.18(0.74)$ & $4.06(0.73)$ & $3.96(0.74)$ & $4.07(0.74)$ \\
\hline FEV1 L & $3.15(0.66)$ & $3.11(0.63)$ & $3.03(0.66)$ & $3.10(0.66)$ \\
\hline Smoking pack-yrs* & $17.6 \quad(23.8)$ & $20.8 \quad(25.6)$ & $22.8 \quad(24.3)$ & 20.4 (24.6) \\
\hline Physical activity $\mathrm{kcal} \cdot \mathrm{week}^{-1 * * *}$ & 2698.6 & 2501.3 & 2039.7 & 2414.0 \\
\hline Alcohol intake $\mathrm{g} \cdot \mathrm{day}^{-1} *$ & $(22.1)$ & $13.6(16.7)$ & $(19.0)$ & $14.8 \quad(19.5)$ \\
\hline
\end{tabular}

Values are shown as mean (SD). +: log-transformed for analysis of variance (ANOVA). FIRI: fasting insulin resistance index; BMI: body mass index; FVC: forced vital capacity; FEV1: forced expiratory volume in one second. Significant difference by ANOVA between fasting insulin tertiles at $*$ : $<<0.05 ; * * *$ : pð0.001.

Residual plots from all regression models were examined for evidence of gross nonlinearity or heteroscedasticity and collinearity diagnostics available in the SAS regression procedure were examined to check for collinearity in all regression models.

\section{Results}

Means and standard deviations for the measurements used in this report are shown in table 1 for all the included subjects and by fasting insulin tertile. ANOVA results showed that these means were significantly different by fasting insulin tertile for age $(\mathrm{p}=0.004)$, BMI $(\mathrm{p}<0.0001)$, WHR $(p<0.0001)$, FVC $(p=0.001)$, alcohol $(p=0.02)$, fasting insulin $(\mathrm{p}<0.0001)$ and FIRI $(\mathrm{p}<0.0001)$, but not height $(\mathrm{p}=0.35)$ or FEV1 $(\mathrm{p}=0.051)$. The overall proportion of subjects with any diagnosis of pre-existing heart disease was $25.2 \%$ and this did not vary significantly by insulin tertile $\left(\chi^{2}=2.3\right.$, d.f. $\left.=2, \mathrm{p}=0.32\right)$.

Both FVC and FEV1 were significantly (all p<0.001) negatively correlated with both FIRI (FVC r $=-0.15$, FEV1 $\mathrm{r}=-0.12$ ) and fasting insulin levels (FVC $\mathrm{r}=-0.14$, FEV 1 $\mathrm{r}=-0.11)$. There were significant correlations between ventilatory function measures and a number of covariates which have been associated with insulin levels in previous studies. In particular, FVC was significantly (all $\mathrm{p}<0.01)$ negatively correlated with age $(\mathrm{r}=-0.44)$, WHR $(\mathrm{r}=-0.17)$ and pack-yrs of smoking $(\mathrm{r}=-0.17)$, confirming that adjustment for the potential confounders of age, height, WHR and smoking may be needed for analyses of the relationship between insulin and ventilatory function.

Results from multiple linear regression models in which FVC and FEV1 were separately regressed on FIRI and fasting insulin in turn are shown in tables 2 and 3. Additional adjustment for BMI, WHR, physical activity, alcohol and pre-existing heart disease made no significant difference to the coefficients on fasting insulin or FIRI from the simpler models, so only the final models are shown. The negative coefficients on fasting insulin and FIRI suggest that subjects with higher insulin levels and greater insulin resistance tended to have significantly lower levels of ventilatory function after adjustment for potential confounders.

Standardized regression coefficients [31] are also presented in tables 2 and 3. These indicate the predicted mean change (in SD units) of the outcome variable, for a mean change of $1 \mathrm{sD}$ in the predictor variable. As expected, variability in height and age were the major determinants of variation in FVC. The effects of variation in measures of insulin resistance were similar to the effects of variation in measures of fat distribution and cigarette smoking, while there was a small additional contribution from alcohol intake. The pattern for FEV1 was similar,

Table 2. - Regression models of log-transformed fasting insulin resistance index (FIRI) predicting ventilatory function with adjustment for potential confounders

\begin{tabular}{|c|c|c|c|c|}
\hline \multirow[b]{2}{*}{ Term } & \multicolumn{2}{|c|}{ Model predicting FVC } & \multicolumn{2}{|c|}{ Model predicting FEV1 } \\
\hline & $\begin{array}{c}\text { Parameter } \\
\text { estimate (p-value) }\end{array}$ & $\begin{array}{c}\text { Standardized } \\
\text { coefficient }\end{array}$ & $\begin{array}{c}\text { Parameter } \\
\text { estimate (p-value) }\end{array}$ & $\begin{array}{c}\text { Standardized } \\
\text { coefficient }\end{array}$ \\
\hline Intercept & $-0.58(0.51)$ & & $-0.15(0.86)$ & \\
\hline $\mathrm{FIRI}^{+}$ & $-0.18(0.0001)$ & -0.14 & $-0.14(0.0001)$ & -0.12 \\
\hline Age yrs & $-0.033(0.0001)$ & -0.34 & $-0.03(0.0001)$ & -0.37 \\
\hline Height $\mathrm{m}$ & $4.80(0.0001)$ & 0.44 & $3.34(0.0001)$ & 0.33 \\
\hline $\mathrm{BMI} \mathrm{kg} \cdot \mathrm{m}^{-2+}$ & $-0.16(0.37)$ & -0.01 & $-0.40(0.02)$ & 0.08 \\
\hline Waist/hip ratio & $-1.91(0.0001)$ & -0.12 & $-1.80(0.0001)$ & -0.13 \\
\hline Smoking pack-yrs & $-0.005(0.0001)$ & -0.15 & $-0.007(0.0001)$ & -0.24 \\
\hline Current smoker & $-0.02(0.87)$ & -0.005 & $-0.05(0.49)$ & -0.02 \\
\hline Physical activity $\mathrm{kcal} \cdot \mathrm{week}^{-1}$ & $0.00002(0.02)$ & 0.06 & $0.000016(0.038)$ & 0.06 \\
\hline Alcohol intake $\mathrm{g} \cdot \mathrm{day}^{-1}$ & $-0.002(0.03)$ & -0.06 & $-0.002(0.05)$ & -0.05 \\
\hline Pre-existing heart disease & $-0.06(0.16)$ & -0.04 & $-0.005(0.91)$ & -0.003 \\
\hline
\end{tabular}

Values are shown as mean (SD). + ${ }^{+}$log-transformed. FVC: forced vital capacity; FEV1: forced expiratory volume in one second; BMI: body mass index. 
Table 3. - Regression models of log-transformed fasting insulin predicting ventilatory function with adjustment for potential confounders

\begin{tabular}{|c|c|c|c|c|}
\hline \multirow[b]{2}{*}{ Term } & \multicolumn{2}{|c|}{ Model predicting FVC } & \multicolumn{2}{|c|}{ Model predicting FEV1 } \\
\hline & $\begin{array}{c}\text { Parameter } \\
\text { estimate (p-value) }\end{array}$ & $\begin{array}{c}\text { Standardized } \\
\text { coefficient }\end{array}$ & $\begin{array}{c}\text { Parameter } \\
\text { estimate (p-value) }\end{array}$ & $\begin{array}{c}\text { Standardized } \\
\text { coefficient }\end{array}$ \\
\hline Intercept & $-0.05(0.96)$ & & $0.28(0.78)$ & \\
\hline Insulin+ & $-0.17(0.0001)$ & -0.12 & $-0.13(0.0008)$ & -0.10 \\
\hline Age yrs & $-0.033(0.0001)$ & -0.34 & $-0.03(0.0001)$ & -0.38 \\
\hline Height $\mathrm{m}$ & $5.02(0.0001)$ & 0.44 & $3.3(0.0001)$ & 0.33 \\
\hline $\mathrm{BMI} \mathrm{kg} \cdot \mathrm{m}^{-2+}$ & $-0.10(0.61)$ & -0.02 & $-0.37(0.03)$ & 0.07 \\
\hline Waist/hip ratio & $-1.80(0.0001)$ & -0.12 & $-1.81(0.0001)$ & -0.13 \\
\hline Smoking pack-yrs & $-0.005(0.0001)$ & -0.15 & $-0.007(0.0001)$ & -0.24 \\
\hline Current smoker & $-0.008(0.92)$ & -0.003 & $-0.07(0.53)$ & -0.02 \\
\hline Physical activity $\mathrm{kcal} \cdot$ week $^{-1}$ & $0.00002(0.02)$ & 0.059 & $0.00002(0.043)$ & 0.05 \\
\hline Alcohol intake $g \cdot d^{-1}$ & $-0.002(0.02)$ & -0.60 & $-0.002(0.054)$ & -0.05 \\
\hline Pre-existing heart disease & $-0.07(0.12)$ & -0.04 & $-0.01 \quad(0.74)$ & -0.009 \\
\hline
\end{tabular}

Values are shown as mean (SD). +: log-transformed. FVC: forced vital capacity; FEV1: forced expiratory volume in one second; BMI: body mass index.

except that life-total cigarette smoking was a more important source of variation than was the case for FVC. Least square adjusted mean FVC by insulin tertile from a generalized linear model varied from $4.13 \mathrm{~L}$ in the lowest tertile to $3.98 \mathrm{~L}$ in the highest tertile.

\section{Discussion}

\section{Main findings}

A significant negative correlation was found between both FVC and FEV1 and indirect measures of insulin resistance in this group of nondiabetic males. Males in the highest tertile of fasting insulin level had a mean unadjusted FVC $220 \mathrm{~mL}$ lower than males in the lowest tertile (table 1). The association between higher fasting insulin and FIRI levels and lower levels of ventilatory function persisted in models that included adjustment for major potential confounders. After adjustment for known potential confounders, the difference in mean FVC between males in the top and bottom tertiles for fasting insulin was $150 \mathrm{~mL}$ or about $3.6 \%$. The extent to which variation in fasting insulin levels was associated with variability in FVC was comparable in magnitude to the effect of lifetotal cigarette smoking.

\section{Limitations}

This sample comprised mature males only, so the generalizability of the findings, particularly to females, is not known. The rates of incident NIDDM were lower in this cohort than in other Caucasian samples. This is at least partially explained by the strict entry criteria, which excluded glucose-intolerant subjects.

In this relatively large-scale study, results of specialized laboratory methods for quantification of insulin resistance were not available. The FIRI has been proposed as a simple, indirect measure of insulin resistance [26], although it has been the subject of some controversy $[32,33]$. In addition, fasting insulin has been recommended for epidemiological studies in nondiabetic subjects since it is highly correlated with the results of the euglycaemic hyperinsuli- naemic clamp method, even in subjects with impaired glucose tolerance [16].

Although the measures available from this relatively large cohort are not ideal, it seems reasonable to interpret the findings as representative of the effects of increasing degrees of insulin resistance. The fact that the present measures of insulin resistance were subject to error compared with the gold-standard measures may have introduced bias into the results. If the errors of measurement were distributed randomly with respect to ventilatory function, then the direction of this bias is likely to have been towards a finding of no association and the results may underestimate the true association as a result. The standardized regression coefficients on FIRI were about $15 \%$ larger than those on fasting insulin, suggesting that, in practice, there is a small gain from using the FIRI.

Excess body fat, particularly when located in the abdomen, is known to influence both insulin resistance [30] and ventilatory function [34]. The analyses presented here were adjusted for both overall obesity and a measure of body fat distribution (waist to hip circumference ratio). It may be argued that inclusion of terms for obesity and fat distribution would result in overadjustment, since these may be on the causal chain for insulin resistance. In practice, adding these terms to the models made no significant difference to the regression coefficients on insulin resistance measures.

The cross-sectional data reported here do not offer insight into the temporal relationship between insulin resistance and ventilatory function. This may be a subject worthy of study using prospective data.

\section{Ventilatory function, glucose intolerance and NIDDM}

A number of previous studies has reported that measures of glucose intolerance may be associated with impaired ventilatory function. Postcarbohydrate challenge (PC) glucose was negatively correlated with FEV1 in an unadjusted analysis from the Honolulu Heart Study [9], while significant unadjusted correlations between FVC and both fasting and PC insulin levels were reported [10] in a later publication from the same cohort. Higher levels of plasma glucose were correlated with lower levels of FVC and FEV1 in the Copenhagen City Heart Study co- 
hort [11]. In nondiabetic subjects, an inverse relationship between fasting plasma glucose and both FVC and FEV1 was reported among males from the Rancho Bernardo cohort, but not among females [12]. None of these analyses was adjusted for body fat distribution, alcohol or physical activity.

In a cross-sectional analysis from the Copenhagen City Heart Study [11], significant ventilatory function impairment was reported among self-reported diabetics treated with diet or oral hypoglycaemic drugs. A greater level of impairment was observed in diabetics using insulin. Among nondiabetic subjects, a significant negative correlation between nonfasting plasma glucose and both FVC and $\mathrm{FEV}_{1}$ was noted. A follow-up study from the same cohort [8] found that the steepest rates of decline in FVC and FEV 1 occurred in those subjects who developed diabetes during the $5 \mathrm{yr}$ follow-up period. Although no measures of insulin were reported, it is likely that those subjects developing NIDDM had the most substantial increases in insulin levels over the follow-up period.

There is general agreement that resistance to the glucose-regulatory actions of insulin precedes glucose intolerance in the natural history of NIDDM [13]. Findings from other studies of a relation between glucose intolerance, NIDDM and ventilatory function appear to be consistent with the association reported here with insulin resistance. The exclusion of diabetics from the subjects reported in this study implies that diminished ventilatory function is associated with elevated insulin resistance long before NIDDM supervenes.

\section{Ventilatory function and cardiovascular disease}

Lower levels of FVC and FEV1 were found in subjects who experienced sudden cardiac death or myocardial infarction than in controls in a follow-up after multiphasic health screening [1]. An increased risk of cardiovascular death, which persisted after adjustment for other cardiovascular risk factors, was found in subjects with lower levels of baseline FVC, during 20 yrs of follow-up in the Framingham study [2]. Higher levels of baseline peak flow rate predicted lower risk of myocardial infarction during 12 yrs of follow-up in a population study of Swedish females [4]. The risk of cardiovascular death was negatively associated with baseline ventilatory function during an average of 6.5 yrs follow-up in the Copenhagen City Heart Study [3]. Although the association is consistent and long recognized, it remains largely unexplained [5].

\section{Interpretation}

A significant negative cross-sectional association between ventilatory function and indirect measures of insulin resistance has been found in nondiabetic subjects from the NAS which persisted after adjustment for known potential confounders. Previous reports have shown that glucose intolerance and NIDDM may be associated with impaired ventilatory function. The results reported here suggest that insulin resistance, which generally precedes the development of glucose intolerance and NIDDM, may be the underlying abnormality.

The finding of lower forced vital capacity and forced expiratory volume in one second in subjects with higher fasting insulin resistance index and fasting insulin is consistent with a role for insulin as one of the mechanisms in the hitherto unexplained relationship between cardiovascular disease risk and decreased ventilatory function, because subjects with higher levels of insulin are at increased risk of coronary heart disease [17]. Insulin resistance, which may initially give rise to hyperinsulinaemia and eventually result in glucose intolerance and noninsulindependent diabetes mellitus (which are themselves wellrecognised risk factors for coronary heart disease), may be the underlying mechanism. However, the means by which ventilatory function is associated with insulin resistance, hyperinsulinaemia and glucose intolerance remain to be elucidated.

\section{References}

1. Friedman G, Klatsky A, Siegelaub M. Lung function and risk of myocardial infarction and sudden cardiac death. $N$ Engl J Med 1976; 294: 1071-1075.

2. Kannel W, Hubert H, Lew E. Vital capacity as a predictor of cardiovascular disease: the Framingham study. Am Heart J 1983; 105: 311-315.

3. Lange P, Nyboe J, Jensen G, Schnohr P, Appleyard M. Ventilatory function impairment and risk of cardiovascular death and of fatal or non-fatal myocardial infarction. Eur Respir J 1991; 4: 1080-1087.

4. Persson C, Bengtsson C, Lapidus L, Rybo E, Thringer G, Wedel H. Peak expiratory flow rate and risk of cardiovascular disease and death: a 12-year follow-up of participants in the population study of women in Gothenburg, Sweden. Am J Epidemiol 1986; 124: 942-948.

5. Beaty T, Cohen B, Newill C, Menkes H, Diamond E, Chen C. Impaired pulmonary function as a risk factor for mortality. Am J Epidemiol 1982; 116: 102-113.

6. Schuyler M, Niewoehner D, Inkley S, Kohn R. Abnormal lung elasticity in juvenile onset diabetes mellitus. Am Rev Respir Dis 1976; 113: 37-41.

7. Wanke T, Formanek D, Auinger M, Popp W, Zwick H, Irsigler K. Inspiratory muscle performance and pulmonary function changes in insulin dependent diabetes mellitus. Am Rev Respir Dis 1991; 143: 97-100.

8. Lange P, Groth S, Mortensen J, et al. Diabetes mellitus and ventilatory capacity: a five year follow-up study. Eur Respir J 1990; 3: 288-292.

9. Marcus E, Buist S, Curb D, et al. Correlates of FEV1 and prevalence of pulmonary conditions in Japanese-American men. Thorax 1988; 138: 1398-1404.

10. Burchfiel C, Curb D, Sharp D, et al. Distribution and correlates of insulin in elderly men: the Honolulu heart program. Arterioscl Thromb Vasc Biol 1995; 15: 2213-2221.

11. Lange P, Groth S, Kastrup J, et al. Diabetes mellitus, plasma glucose and lung function in a cross-sectional population study. Eur Respir J 1989; 2: 14-19.

12. Barrett-Connor E, Frette C. NIDDM, impaired glucose tolerance, and pulmonary function in older adults. Diabetes Care 1996; 19: 1441-1444.

13. Hamman R. Genetic and environmental determinants of non-insulin-dependent diabetes mellitus (NIDDM). Diabetes Metab Rev 1992; 8: 287-338.

14. Saad MF, Knowler WC, Pettitt DJ. A two-step model for development of noninsulin dependent diabetes. Am J Med 1991; 90: 229-235.

15. Nijpels G, Popp-Snijders C, Kostense PJ, Bouter LM, Heine RJ. Cardiovascular risk factors prior to the devel- 
opment of non-insulin dependent diabetes mellitus in persons with impaired glucose tolerance: the Hoorn study. $J$ Clin Epidemiol 1997; 50: 1003-1009.

16. Laakso M. How good a marker is insulin level for insulin resistance? Am J Epidemiol 1993; 137: 959-965.

17. Perry I, Wannamethee SG, Whincup P, Shaper AG, Walker M, Alberti KG. Serum insulin and incident coronary heart disease in middle-aged British men. Am J Epidemiol 1996; 144: 224-234.

18. DeFronzo R, Ferrannini E. Insulin resistance: a multifaceted syndrome responsible for NIDDM, obesity, hypertension, dyslipidemia and atherosclerotic cardiovascular disease. Diabetes Care 1991; 14: 173-194.

19. Bell B, Rose C, Damon H. The normative aging study: an interdisciplinary and longitudinal study of health and aging. Aging Hum Dev 1972; 3: 5-17.

20. Willett W. Anthropometric measures and body composition. In: MacMahon B, ed. Nutritional Epidemiology. 1st Edn. Monographs in Epidemiology and Biostatistics, Vol. 15. New York, Oxford University Press, 1990.

21. Paffenbarger R, Hyde R, Weng A, Hsieh C. Physical activity, all-cause mortality and longevity of college alumni. N Engl J Med 1986; 314: 605-613.

22. Willett W, Sampson L, Stampfer M, et al. Reproducibility and validity of a semiquantitative food frequency questionnaire. Am J Epidemiol 1985; 122: 51-55.

23. Sampson L. Food frequency questionnaires as a research instrument. Clin Nutr 1985; 4: 171-178.

24. American Thoracic Society. Standardization of spirometry. Am Rev Respir Dis 1979; 119: 831-838.
25. National Diabetes Data Group. Classification and diagnosis of diabetes mellitus and other categories of glucose intolerance. Diabetes 1979; 28: 1039-1057.

26. Duncan MH, Singh BM, Wise PH, Carter G. A simple measure of insulin resistance. Lancet 1995; 346: 120 121.

27. Boyko E, Leonetti D, Bergstrom R, Fujimoto W. Fasting insulin levels underestimates risk of non-insulin dependent diabetes mellitus due to confounding by insulin secretion. Am J Epidemiol 1997; 145: 18-23.

28. Schoenberg J, Beck G, Bouhuys A. Growth and decay of pulmonary function in healthy blacks and whites. Respir Physiol 1978; 33: 367-393.

29. Ronnemaa T, Ronnemaa E, Puukka P, Pyorala K, Laakso M. Smoking is independently associated with high plasma insulin levels in nondiabetic men. Diabetes Care 1996; 19: 1229-1232.

30. Bjorntorp P. The regulation of adipose tissue distribution in humans. Int J Obesity 1996; 20: 291-302.

31. Rosner B. Fundamentals of Biostatistics. Boston, MA, Duxbury Press, 1986.

32. Del Prato S, Pozzilli P. FIRI: fasting or false insulin resistance index ? Lancet 1996; 347: 132.

33. Cleland SJ, Petrie JR, Morris AD, Ueda S, Dorrian CA, Connell JMC. FIRI: a fair insulin resistance index. Lancet 1996; 347: 770.

34. Lazarus R, Sparrow D, Weiss ST. Effects of obesity and fat distribution on ventilatory function. Chest 1997; 111: 891-898. 